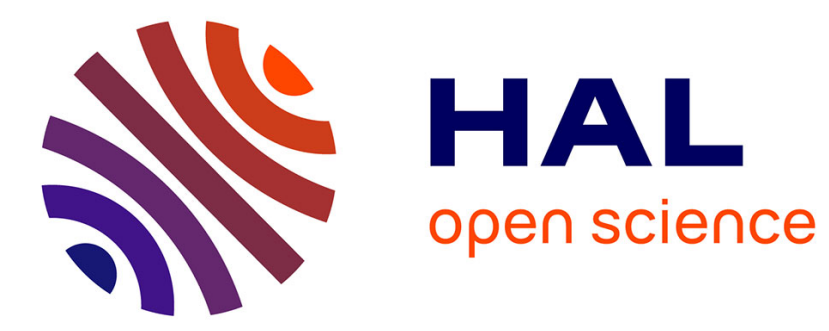

\title{
Linearized potential vorticity mode and its role in transition to baroclinic instability
}

Alexandre Pieri, Claude Cambon, Fabien S. Godeferd, Abdelaziz Salhi

\section{To cite this version:}

Alexandre Pieri, Claude Cambon, Fabien S. Godeferd, Abdelaziz Salhi. Linearized potential vorticity mode and its role in transition to baroclinic instability. Physics of Fluids, 2012, 24, pp.076603. 10.1063/1.4731294 . hal-00780220

\section{HAL Id: hal-00780220 \\ https://hal.science/hal-00780220}

Submitted on 8 Apr 2016

HAL is a multi-disciplinary open access archive for the deposit and dissemination of scientific research documents, whether they are published or not. The documents may come from teaching and research institutions in France or abroad, or from public or private research centers.
L'archive ouverte pluridisciplinaire HAL, est destinée au dépôt et à la diffusion de documents scientifiques de niveau recherche, publiés ou non, émanant des établissements d'enseignement et de recherche français ou étrangers, des laboratoires publics ou privés. 


\title{
Linearized potential vorticity mode and its role in transition to baroclinic instability
}

\author{
A. B. Pieri, ${ }^{1, \text { a) }}$ C. Cambon, ${ }^{1}$ F. S. Godeferd, ${ }^{1}$ and A. Salhi ${ }^{2}$ \\ ${ }^{1}$ Laboratoire de mécanique des fluides et d'acoustique, Ecole Centrale de Lyon, \\ 36 avenue Guy de Collongue, 69134 Écully cedex Lyon, France \\ ${ }^{2}$ Département de physique, faculté des sciences de Tunis, Université Tunis El Manar, \\ 1060 Tunis, Tunisia
}

(Received 10 January 2012; accepted 22 May 2012; published online 9 July 2012)

\begin{abstract}
In addition to the effect of stable stratification and uniform shear in turbulent flows, a case which has already been modelled using linear approaches (rapid distortion theory) and direct numerical simulations, we introduce the effect of uniform rotation. Assuming the rotation axis to be vertical, and aligned with the gradients of density and mean velocity, an enslaved horizontal stratification is forced and baroclinic instability can occur. In that case, the linear analysis shows an extension of the unstable domain up to a gradient Richardson number $R i$ of 1 for $k_{x}=0$ modes. First, we extend this asymptotic analysis at $t \rightarrow \infty$ to $k_{x} \neq 0$ modes. We show that these modes lead asymptotically to oscillating bounded solutions whose frequency depend only on the rotation number $R=1 / R o$. The analysis of the transition to baroclinic instability is then completed with a discussion of the role of $k_{x} \approx 0$ modes-small streamwise wavenumbers - and of the importance of coupling with the potential vorticity mode $u^{\left(\Omega_{p o t}\right)}$ or Ertel's mode. The latter is shown to be determinant for dramatic transient growth at intermediate times. The structure of the stratified, sheared, rotating flow is predicted using a stochastic linear approach (referred to as kinematic simulation) to complete the rapid distortion theory analysis. (c) 2012 American Institute of Physics. [http://dx.doi.org/10.1063/1.4731294]
\end{abstract}

\section{INTRODUCTION}

The baroclinic context is characterized by a superposition of three coupled phenomena: (a) the Coriolis force, caused by the Earth's rotation in geophysical flows; (b) stable stratification due to density gradients in the atmosphere, which lead to buoyancy forces in the vertical direction; and (c) large vertical velocity gradients, e.g., at the altitude of the tropopause in atmospheric flows, in the form of jet streams, which, in first approximation, are modelled by homogeneous shear. Linear theory, or rapid distortion theory (RDT), was applied to this problem by Salhi and Cambon. ${ }^{1}$ It was shown ${ }^{2}$ that the gyroscopic torque resulting from the misalignment of system vorticity, in the vertical direction, and shear-induced vorticity, in the spanwise direction (see Figure 1(b)), can exactly be balanced by an additional density gradient in the horizontal direction. This mechanism is close to the "geostrophic front adjustment" in real geophysical flows, ${ }^{3}$ and allows to use the simplified formalism of RDT for homogeneous turbulence (and direct numerical simlations presented in European Turbulence Conference, 13th edition, Warsaw, 2011 (Ref. 4)). The additional mean density gradient tilts the mean isopycnal surfaces with respect to the horizontal direction, so that baroclinic instability is triggered. For a mean flow characterized by a shear rate $S$, a vertical stabilizing mean buoyancy gradient resulting in a constant Brunt-Väisälä frequency $N$, and a Coriolis parameter $f$ in the $f$-plane approximation, the baroclinicity parameter $\varepsilon_{B}=f S / N^{2}$ is also the above-mentioned angle of isopycnals and is a crucial parameter for the baroclinic instability.

a) Author to whom correspondence should be addressed. Electronic mail: alexandre.pieri@ec-lyon.fr. 


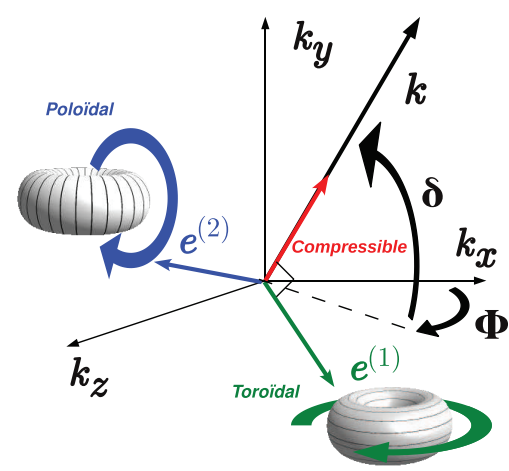

(a)

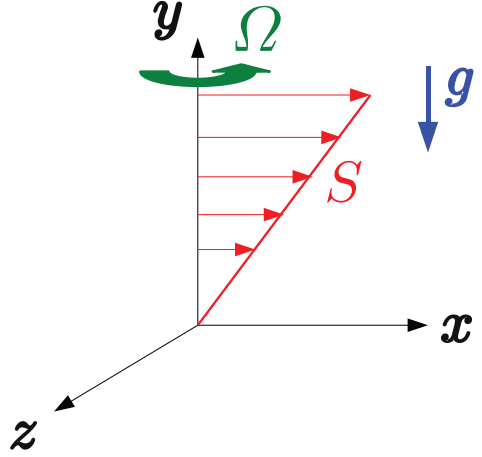

(b)

FIG. 1. (a) The Craya-Herring frame: the divergence-free condition is implicitly imposed by locally projecting on the solenoidal base formed by planes perpendicular to each wavevector $\boldsymbol{k}$. The compressible mode would appear along $\boldsymbol{k}$ for compressible flows. (b) The baroclinic configuration, with vertical rotation $\Omega$ and gravity $g$, and uniform vertical mean shear $S$.

The other relevant independent external parameter is the gradient Richardson number $R i=N^{2} / S^{2}$. Linear analysis is here referred to as RDT, following the nomenclature used in engineering and geophysics by Salhi and Cambon, ${ }^{1}$ but there exist many studies based on the same formalism in other disciplines that use different terminologies, as in Mamatsashvili et al. ${ }^{5}$ using the shearing sheet approximation from astrophysicists. The reader is referred to Salhi et al. ${ }^{6}$ for a survey of this linear theory in engineering, applied mathematics and astrophysics.

RDT can be extended towards fully nonlinear direct numerical simulations (DNS) in the context of statistically homogeneous turbulence. For this purpose, pseudo-spectral DNS in deformed coordinates was introduced by Rogallo. ${ }^{7}$ Without Coriolis force, and therefore without baroclinic effects, the combined effects of pure plane shear and vertical stabilizing density stratification were studied by DNS using this numerical technique or another, ${ }^{8-10}$ Large Eddy Simulations were used to reach higher Reynolds number turbulence, ${ }^{11}$ and results from experimental studies in wind tunnels are available. ${ }^{12,13}$ Linear analysis was also employed that can be used to predict the short time behavior of low potential energy stably stratified sheared turbulence,,${ }^{14}$ but it also can be related to classical hydrodynamic stability analyses in the same context or with non-uniform shear. ${ }^{15}$

In the numerical simulations by Jacobitz et al. various results on statistics are presented. It appears that marginal instability, with nonlinear sustaining of the turbulent kinetic energy, was shifted from negative Richardson numbers (unstable stratification) up to a small positive value $R i \sim 0.15$. Another well-known threshold Richardson number is $1 / 4$, but this is found, in the linear limit, in the presence of base vertical profile with inflexional point, therefore for a non-uniform shear in the vertical direction. ${ }^{16,17}$

In the extended context of stably stratified sheared flows with rotation, less experimental or numerical results are available. The main contribution of Salhi and Cambon ${ }^{1}$ was to demonstrate that the domain of exponential instability was shifted to $R i=1$ with additional Coriolis force and additional enslaved horizontal stratification. This result is supported by analytical solutions of the general RDT problem at $k_{x}=0$, with a neutral curve in terms of both the Richardson number and the inclination of the wave number of the disturbance. Further numerical investigation by Simon ${ }^{18}$ and Pieri et al., ${ }^{4}$ with the linear model and nonlinear simulations, suggested that the domain of important, even transient, growth rate is concentrated near the $k_{x}=0$ manifold.

New elements on the strong transient growth of perturbations normally located in the "stable" range of linear stability, were brought out following Ref. 5, and can be explained as follows. Exponential growth is not prescribed in RDT as in conventional modal stability analysis in which spatio-temporal disturbances are assumed of the form $\boldsymbol{u}(\boldsymbol{x}) \exp \omega t$. RDT spatial modes are defined differently, as advected Fourier modes, but with temporal variation that result from solving a linear system of Ordinary Differential Educations. In this system, the eigenvectors are often time-dependent and nonorthogonal, as a result of the non-self-adjointedness of the linear operators. ${ }^{19}$ In all cases 
where distortion results from the pure plane shear, even with additional Coriolis or buoyancy effects, two cases must be distinguished. At $k_{x}=0$, which is called the axisymmetric mode in Refs. 5 and 20 , the wave-vector is no longer time-dependent, and no important transient growth is found. Only if $k_{x}$ is nonzero, even small, does the time-dependency of the wave-vector reflect the advection by the mean shear flow. This advection term is responsible for the non-self-adjointness of the linear operator and thus of non-modal growth. A last ingredient is the generalized "wave-vortex" decomposition suggested by Tevzadze et al..$^{21}$ the conservation of potential vorticity yields a linear invariant. Mode coupling between this constant "vortex" mode and the rest of the perturbation field is essential for transient growth, but only at $k_{x} \neq 0$. At $k_{x}=0$, in the exponentially stable parametric model, one only recovers a superposition of the constant "vortex" mode with oscillating "wave" modes as in the shearless case.

Our consistent RDT approach can be described with the following five stages:

1. Definition of a mean (or base) flow which is an exact solution of Euler-Boussinesq equations. This "admissibility condition" (from A. C. C. Craik) permits to balance the gyroscopic torque in a physical way-without the addition of adhoc compensating terms in the equations-by looking at the conservation of mean absolute vorticity derived from the Euler equations.

2. Decomposition of the fluctuating flow in terms of advected Fourier modes with time-dependent wave-vectors. These modes are called "Kelvin modes" (in Applied Mathematics, probably following H. K. Moffatt ${ }^{22}$ in RDT) and "shear waves" (in Astrophysics) and corresponds to "Rogallo space" (in Engineering). In addition, the fluctuating velocity modes are split using the Craya-Herring frame of reference, resulting in a minimal number of two solenoidal components.

3. The linear solution is generated by a deterministic Green's function, applied to the velocity/buoyancy fluctuating field, for arbitrary initial data and possibly including arbitrary forcing, prior to any calculation of turbulent statistics.

4. Classical conservation of potential vorticity (PV) is applied. In the "RDT" context, this results in an invariant of the motion, as the linearized absolute potential vorticity, in which the vorticity of the mean shear is involved.

5. Predictions of both random turbulence realizations of the velocity field, as in Kinematic Simulation (KS) (Ref. 23) and RDT-based KS, and of statistical quantities, as in conventional RDT.

Formally, the previous item 2 reduces the basic linear problem to a third-rank system of ordinary equations with time-dependent coefficients. The fluctuating field comprises five components in physical space: three components for the velocity, one for the buoyancy and one for the pressure. It reduces to three components in Fourier space when using the decomposition into toroidal and poloidal components-also called the Craya-Herring decomposition-along with the fluctuating density. Each of the previous components are associated respectively with poloidal, toroidal, and potential energy. The rank of the system is eventually reduced to a non-homogeneous system of two equations, thanks to PV conservation mentioned in item 4. In addition to the exact equations, which are given here, a generalized "vortex-wave" 24 decomposition is derived, which provides a physical interpretation.

Rapid distortion theory is often used to provide results on the asymptotic behavior of the flow (the rapid short time limit is rediscussed in Sec. V). For instance, Moffatt ${ }^{22}$ exposes a comprehensive study of long time linear dynamics of pure shear flows. Salhi and Cambon ${ }^{1}$ and Timoumi and $\mathrm{Salhi}^{25}$ use the same RDT approach to predict asymptotic results on the kinetic energy growth rate for baroclinic turbulence. In the present paper, we also use RDT for transient growth analysis. We first recall briefly how asymptotic results are derived. In particular, we insist on the dominance of $k_{x}=0$ modes at infinite nondimensionalized time St. In the second part, we show that, at large but finite times St, RDT predicts another trend. Including Ertel's potential vorticity in the RDT model, we show how the initial distribution of PV affects the transient behavior of the flow and selects the dominant modes. This analysis give a complementary geophysical interpretation to the work by Mamatsashvili et al., ${ }^{5}$ in which the role of $k_{x} \approx 0$ (nonsymmetric perturbations in Ref. 5 is indeed determinant for transition to baroclinic instability, but does not explain itself why 
the transient growth can be zero for some modes). The latter modes will be referred to as wave modes in the present article. We therefore show that RDT coupled to a vortex-wave decomposition can answer this question.

The paper is organized as follows. Section II recalls the equations of motion with a brief introduction to potential vorticity. In Sec. III we present the linear model in the Craya-Herring Fourier space. From this model we recover asymptotic results on the most unstable modes with comparison to DNS in Sec. IV, and the model based on RDT coupled with Kinematic Simulation is presented in Sec. V. Section VI presents the main results about the transient growth of $k_{x} \approx 0$ modes, with analytical details exposed in Appendixes A and C. We finally conclude in Sec. VII.

\section{EQUATIONS OF MOTION}

We consider an incompressible fluid with a vertical gradient of density placed in a gravity field of amplitude $g$ and in a frame rotating at a rate $\Omega$ along the vertical axis $z$. The following equations are written in the rotating frame, such that only the Coriolis force appears explicitly. The flow is submitted to a mean uniform shear $S$ along the vertical direction such that the mean velocity is $\overline{\boldsymbol{u}}=S y \boldsymbol{e}_{x}$ where $\boldsymbol{e}_{x}$ is the unit vector along the $x$-axis. For the flow to be homogeneous, we assume a linear dependency of the mean velocity field with the spatial coordinates. We denote mean background quantities by an overbar.

Due to the simultaneous presence of shear and rotation, geostrophic adjustment produces a spanwise density gradient that compensate mean vorticity production in the streamwise direction, as illustrated by Eq. (1) for the streamwise component of the mean vorticity:

$$
\begin{aligned}
& \omega=\overline{\boldsymbol{\omega}}+\boldsymbol{\omega} \\
& =\nabla \times \overline{\boldsymbol{u}}+\nabla \times \boldsymbol{u}, \\
& \frac{\partial \bar{\omega}_{x}}{\partial t}-S f=-\frac{\partial \bar{b}}{\partial z} .
\end{aligned}
$$

In this equation, $S f$ is the magnitude of the gyroscopic torque, that results from misalignment of shear-induced mean vorticity (spanwise) with system vorticity (vertical). If the gradient of $\bar{b}$ is only vertical, a spurious component of streamwise vorticity appears. In order to conserve a stationary mean flow solution of Euler-Boussinesq equations, admissibility condition then implies that

$$
\frac{\partial \bar{b}}{\partial z}=S f \triangleq \varepsilon_{B} N^{2}
$$

The corresponding mean buoyancy field $\bar{b}$ at hydrostatic equilibrium is thus

$$
\bar{b}=N^{2} y+\varepsilon_{B} N^{2} z
$$

and its associated gradient

$$
\nabla \bar{b}=N^{2} \boldsymbol{e}_{y}+\varepsilon_{B} N^{2} \boldsymbol{e}_{z}
$$

We consider a Boussinesq fluid, i.e., temperature fluctuations are assumed to be small compared to the mean temperature. The fluctuating density field $\rho$ is linked to the fluctuating temperature field $T$ through the thermal expansion coefficient $\beta$ by the relation $\rho=\beta T \rho_{0}$. The Brunt-Väisälä frequency $N$ is then $N=\left(-g \partial_{y} \bar{\rho} / \rho_{0}\right)^{1 / 2}$ or $N=\left(g \beta \partial_{y} \bar{T}\right)^{1 / 2}$ if expressed using the mean temperature gradient. The buoyancy field $b$ is then defined as the magnitude of the buoyancy force and equals $b=-g(\rho$ $\left.-\rho_{0}\right) / \rho_{0}$ or $b=g\left(T-T_{0}\right) / T_{0} \cdot{ }^{26,27}$ The Coriolis frequency $f=2 \Omega$ is twice the rotation rate. A sketch of the baroclinic configuration is given in Fig. 1(b). The nonlinear conservation equations for the fluctuating velocity and buoyancy fields are

$$
\frac{\partial \boldsymbol{u}}{\partial t}+\overline{\boldsymbol{u}} \cdot \nabla \boldsymbol{u}+\boldsymbol{u} \cdot \nabla \overline{\boldsymbol{u}}+\boldsymbol{u} \cdot \nabla \boldsymbol{u}=-\nabla p^{*}+v \nabla^{2} \boldsymbol{u}-2 \boldsymbol{\Omega} \times \boldsymbol{u}+b \boldsymbol{e}_{y},
$$




$$
\nabla \cdot \boldsymbol{u}=0
$$

$$
\frac{\partial b}{\partial t}+\boldsymbol{u} \cdot \nabla b+\overline{\boldsymbol{u}} \cdot \nabla b=-\boldsymbol{u} \cdot \nabla \bar{b}+\chi \nabla^{2} b,
$$

where the centrifugal force has been included in the pressure term according to $\nabla p^{*}=\nabla p+\boldsymbol{\Omega}$ $\times(\boldsymbol{\Omega} \times \boldsymbol{r})$. Here, $\chi$ is the thermal diffusivity and $v$ is the kinematic viscosity.

The inviscid linear approximation is obtained by keeping only first-order terms for the fluctuating field and by neglecting the dissipation term in the above equations. The set of linearized inviscid Boussinesq equations is therefore

$$
\begin{aligned}
\frac{\partial \boldsymbol{u}}{\partial t}+S y \boldsymbol{e}_{x} \cdot \nabla \boldsymbol{u}+\boldsymbol{u} \cdot \nabla\left(S y \boldsymbol{e}_{x}\right) & =-\nabla p^{*}-2 \boldsymbol{\Omega} \times \boldsymbol{u}+b \boldsymbol{n}, \\
\nabla \cdot \boldsymbol{u} & =0, \\
\frac{\partial b}{\partial t}+S y \boldsymbol{e}_{x} \cdot \nabla b & =-\boldsymbol{u} \cdot \nabla\left(N^{2} y+\varepsilon_{B} N^{2} z\right) .
\end{aligned}
$$

These equations yield an important Lagrangian invariant in geophysics, the potential vorticity.

The conservation of potential vorticity $(\nabla \bar{b}+\nabla b) \cdot(\bar{\omega}+\omega)$ is a well-known consequence of Ertel's theorem applied to the Boussinesq equations. ${ }^{28,29}$

If the velocity field is decomposed in the mean isopycnal frame of reference as

$$
\boldsymbol{u}=u_{\perp} \frac{\nabla \bar{b}}{\|\nabla \bar{b}\|}+\boldsymbol{u}_{\pi}
$$

then $\boldsymbol{u}_{\pi}$ is the part of the velocity field that lies on the isopycnal (isodensity) surfaces. An interpretation of this decomposition in terms of wave-vortex modes is rediscussed in Sec. VI. It expresses the conservation of vorticity $\boldsymbol{\omega}$ projected on the normal $\boldsymbol{n}_{\rho}=\nabla \rho /\|\nabla \rho\|$ to iso-density surfaces along the flow (or isopycnals): $D_{t}\left(\boldsymbol{\omega} \cdot \boldsymbol{n}_{\rho}\right)=0$ where $D_{t}$ is the substantial derivative $D_{t}=\partial_{t}+\boldsymbol{u} \cdot \nabla$. Historically, Rossby was the first to introduce the potential vorticity, ${ }^{29}$ while the conservation theorem was derived two years later by Ertel. ${ }^{28}$ A similar derivation of Eq. (11) can be found in physical space for stably stratified flows with mean velocity shear. ${ }^{30}$ Once the inviscid Boussinesq equations for the fluctuating field are linearized, the same result is obtained by normalizing or not the buoyancy gradient and we have directly that

$$
\boldsymbol{\omega} \cdot \nabla \bar{b}=(\nabla \times \boldsymbol{u}) \cdot \nabla \bar{b}=\left(\nabla \times \boldsymbol{u}_{\pi}\right) \cdot \nabla \bar{b} .
$$

Applied to the previous linearized equations (other nonlinear variants exist), this conservation property can be written as $(\nabla \bar{b} \cdot \omega+\bar{\omega} \cdot \nabla b)+\bar{\omega} \cdot \nabla \bar{b}$, and is the sum of

$$
\bar{\omega} \cdot \nabla \bar{b}=f S^{2}(R i-1),
$$

for the mean background contribution and

$$
\nabla \bar{b} \cdot \omega+\overline{\boldsymbol{\omega}} \cdot \nabla b=f \partial_{y} b-S \partial_{z} b+\omega_{y} N^{2}+\varepsilon_{B} N^{2} \omega_{z},
$$

for the linearized contribution.

The linearized limit is equivalent to considering that isopycnal surfaces are planes, whereas its nonlinear counterpart extends to wavy isopycnal surfaces.

In the linear model detailed in Sec. III, Eq. (14) means that the rank of the linear system can be reduced to two by linear combination of the unknowns following Eq. (14).

Equations (8)-(10) and (14) are the basic equations for our RDT analysis. The tensorial linear system is obtained by introducing a Fourier decomposition for the fluctuating fields $\boldsymbol{u}$ and $b$. Details of its derivation are given in Appendix A. In Sec. III, we present the RDT model equations that describe the evolution of each spectral mode associated with the wavevector $\boldsymbol{k}$. 


\section{RAPID DISTORTION THEORY FOR A BAROCLINIC FLOW}

The analysis is carried out by decomposing the velocity field in a polar-spherical frame based on the vertical axis direction, a.k.a. the Craya-Herring frame. Since the divergenceless property amounts to $\hat{\boldsymbol{u}} \cdot \boldsymbol{k}=0$ in Fourier space, the velocity field is projected on the solenoidal toroidalpoloidal basis relative to each wavevector $\boldsymbol{k}$ defined in spherical coordinates by its modulus $k$ and orientation angles $\delta$ and $\phi$. The colatitude is $\delta \in[-\pi / 2, \pi / 2]$ and the longitude $\phi \in[0,2 \pi]$ are respectively the co-latitude and longitude (see Fig. 1(a)). The corresponding two toroidal and poloidal velocity components are denoted $u^{(1)}$ and $u^{(2)}$. After Fourier transformation, the above linear system of equations is projected onto the unit vectors of the Craya-Herring frame, and yield the following equations for the Fourier coefficients $\hat{\boldsymbol{u}}$ and $\hat{b}$ of the fluctuating velocity and buoyancy fields:

$$
\frac{d}{d t}\left[\begin{array}{l}
u^{(1)} \\
u^{(2)} \\
u^{(3)}
\end{array}\right]=S\left[\begin{array}{ccc}
0 & \cos \delta \sin \phi+\varepsilon_{B} R i \sin \delta & 0 \\
-\varepsilon_{B} R i \sin \delta & \sin \delta \cos \delta \cos \phi & -R i \cos \delta \\
-\varepsilon_{B} \cos \phi & \cos \delta+\varepsilon_{B} \sin \delta \sin \phi & 0
\end{array}\right]\left[\begin{array}{l}
u^{(1)} \\
u^{(2)} \\
u^{(3)}
\end{array}\right] .
$$

The variable $u^{(3)}$ is the buoyancy mode rescaled as a velocity as $u^{(3)}=S / N^{2} \hat{b}$. While $k_{x}$ and $k_{z}$ are unchanged and retain their initial value, the advection equation for the vertical component $k_{y}$ of the wavevector $\boldsymbol{k}$ is

$$
\frac{d k_{y}}{d t}=-S k_{x},
$$

which can also be restated in terms of the latitude $\delta$ as

$$
\frac{d \delta}{d t}=S \cos ^{2} \delta \cos \phi
$$

It is worth noting that the solutions of the previous linear system (15) only depend on the orientation of the wavevector $\boldsymbol{k}$ in spectral space and not on its modulus $k$. This permits to perform the stability analysis only focusing on the two spherical angles $(\delta, \phi)$. The predictions obtained through Eq. (15) are decomposed in three sets presented in Secs. IV and VI. Firstly, in Sec. IV A we recall asymptotic results for $k_{x}=0$ modes corresponding to $\phi= \pm \pi / 2$. Secondly, in Sec. IV B we extend this analysis to $k_{x} \neq 0$ modes or $\phi \neq \pm \pi / 2$ modes. Finally, in Sec. VI, we present results on transitional states for modes with $\phi \approx \pi / 2$, based on both the RDT analysis and the KS model.

\section{ASYMPTOTIC ANALYSIS VIA RDT}

\section{A. The particular case of $k_{x}=0$ modes}

Setting $\phi= \pm \pi / 2$ in the system of Eq. (15) we obtain

$$
\frac{d}{d t}\left[\begin{array}{l}
u^{(1)} \\
u^{(2)} \\
u^{(3)}
\end{array}\right]=S\left[\begin{array}{ccc}
0 & \pm \cos \delta+\varepsilon_{B} R i \sin \delta & 0 \\
-\varepsilon_{B} R i \sin \delta & 0 & -R i \cos \delta \\
0 & \cos \delta \pm \varepsilon_{B} \sin \delta & 0
\end{array}\right]\left[\begin{array}{l}
u^{(1)} \\
u^{(2)} \\
u^{(3)}
\end{array}\right]
$$

and the advection equation (17) for $\delta$ becomes

$$
\frac{d \delta}{d t}=0
$$

Therefore, the set of wavevectors with $k_{x}=0$ or equivalently $\phi= \pm \pi / 2$ is unchanged by advection by the mean shear. The equation for the poloidal component $u^{(2)}$ becomes

$$
\frac{d^{2} u^{(2)}}{d t^{2}}=-S^{2} R i\left[\left(\varepsilon_{B} \sin \delta \pm \cos \delta\right)^{2}+\varepsilon_{B}^{2}(R i-1) \sin ^{2} \delta\right] u^{(2)} .
$$

Let us note $\omega_{0}$ the pulsation defined by

$$
\omega_{0}^{2}=S^{2} R i\left[\left(\varepsilon_{B} \sin \delta \pm \cos \delta\right)^{2}+\varepsilon_{B}^{2}(R i-1) \sin ^{2} \delta\right],
$$


which defines minus the growth rate of $u^{(2)}$ in Eq. (20). For $R i>1$, the solutions are stable since $\omega_{0}^{2}>0$. They exhibit bounded oscillations at frequency $\omega_{0}$. In the particular case $R i=1$ (marginal case), the solutions can be unstable and exhibit linear growth with time if $\omega_{0}=0$. This happens for $\delta=\pi / 2 \pm$ atan $\varepsilon_{B}$, meaning that the wavevector $\boldsymbol{k}$ is parallel to the baroclinic slope imposed by the mean density gradients. Finally, if $R i<1$, the solutions can be unstable and have exponential growth with time. This completes the classification of $k_{x}=0$ modes. In order to show that these modes are linearly the most unstable ones, we study in Sec. IV B the case of modes with $k_{x} \neq 0$, and their long-time behavior.

\section{B. The asymptotic bounded state of $k_{x} \neq 0$ modes}

In that case, the advection equation (17) for the wavevector colatitude angle $\delta$ is not trivial any more, and can be solved by means of variable separation in the particular case $\delta \neq \pm \pi / 2$. Using the initial condition $\delta(t=0)=\delta_{0}$, this yields

$$
\delta(t)=\operatorname{atan}\left(\tan \delta_{0}-t S \cos \phi\right) .
$$

It shows that when $\phi \neq \pm \pi / 2$

$$
\lim _{t \rightarrow \infty} \delta(t)= \pm \frac{\pi}{2}
$$

with the consequence that the coefficient of the Kelvin-Moffatt matrix in the system (15) are asymptotically constant. The sign in the limit depends only on the sign of $\cos \phi$. The linear system at infinite time is equivalent to

$$
\frac{d}{d t}\left[\begin{array}{l}
u^{(1)} \\
u^{(2)} \\
u^{(3)}
\end{array}\right]=S\left[\begin{array}{ccc}
0 & \varepsilon_{B} R i \sin \delta & 0 \\
-\varepsilon_{B} R i \sin \delta & 0 & 0 \\
-\varepsilon_{B} \cos \phi & \varepsilon_{B} \sin \delta \sin \phi & 0
\end{array}\right]\left[\begin{array}{l}
u^{(1)} \\
u^{(2)} \\
u^{(3)}
\end{array}\right],
$$

and the asymptotic equation for the poloidal component $u^{(2)}$ becomes

$$
\frac{d^{2} u^{(2)}}{d t^{2}}=-f^{2} u^{(2)}
$$

This last result is particularly interesting. First, it shows that the solutions for $k_{x} \neq 0$ are bounded asymptotically. It confirms that $k_{x}=0$ modes are linearly the most unstable ones. Secondly, it shows that the asymptotic state of modes $k_{x} \neq 0$ depends only on the Coriolis frequency $f$ (see Eq. (25)). The void third column in the matrix of Eq. (24) shows also that, asymptotically, buoyancy and kinetic modes are linearly decoupled. However, all the previous asymptotic analysis is based on a single Fourier mode analysis, but do not provide information on the behavior of a general field consisting of many general Fourier modes, for which the analytical solution is not available. We therefore consider a velocity field built as the superposition of random sorted Fourier modes. It can be considered as a synthetic model for turbulence. Its simplicity notwithstanding, this Kinematic simulation model has provided several good predictions for the statistics of turbulent flows, since its first introduction. ${ }^{23}$ Although the prescribed synthetic field is not a solution of the Navier-Stokes equations, its statistical properties are close to the ones obtained by DNS. Based on the RDT equations (15) and (16), we build such a stochastic kinematic simulation model as an extension of the initial one for isotropic turbulence by. ${ }^{23}$ The coupled RDT-KS model is presented in Sec. V.

\section{RDT-BASED KINEMATIC SIMULATION MODEL}

In this section, we study a baroclinic flow around its hydrostatic equilibrium - when the mean pressure balances baroclinic effects - using a linear synthetic turbulence model in which linear RDT dynamics is incorporated. The use of such a model makes sense when nonlinearities are negligible, i.e., for low turbulence intensities. In geophysical turbulence, the Reynolds number reaches values around $10^{8}$ - with length scales ranging from $\sim 10^{-1} \mathrm{~m}$ up to $10^{5} \mathrm{~m}$-so that there is no hope to capture the complete physics using Kinematic Simulation. However, the dynamics of small scales 
of turbulence in a geophysical baroclinic flow-with a sufficiently small local Reynolds number defining some cut-off length scale $\eta_{K S}$ — can be described with good accuracy by such a model especially if the linear external effects are dominant. Unresolved scales $\eta<\eta_{K S}$ in a large scale model are then mimicked by a stochastic process: the small scales are modelled by some initial random fluctuations which then evolve according to the linearized equations. Statistical convergence is reached by averaging the results over several statistical events. Some applications and results obtained using KS can be found in the litterature, ${ }^{23,31,32}$ with comparisons to DNS particularly for high order Lagrangian moments. ${ }^{33}$ This model will be well suited for example for modelling small scales in the lower baroclinic troposhpere (also called middle atmosphere in the geophysical community). The ability of KS to predict Lagrangian statistics such as plankton dispersion ${ }^{34}$ augurs well for its application to aerosol dispersion in the lower troposphere for example, which is known to affect drastically the radiative budget of the Earth's atmosphere, provided that the flow remains a single phase flow. Our Kinematic Simulation model is based on a stochastic definition of the velocity and buoyancy fields. We define both of these field as a superposition of random Fourier modes, each mode amplitude and wavevector being a solution of the RDT equations (see also Cambon et al. ${ }^{35}$ ). In that, the KS method presented here can be seen as a stochastic resolution method for the differential system (15) with respect to a deterministic resolution based on a fixed grid of discretized wavevectors in spectral space. In KS, we build the fluctuating fields as the superposition of $N$ base modes

$$
\begin{aligned}
& \boldsymbol{u}(\boldsymbol{x}, t)=\sum_{n=1}^{N} \hat{\boldsymbol{u}}_{n}(t) e^{I \boldsymbol{k}_{n}(t) \cdot \boldsymbol{x}}, \\
& b(\boldsymbol{x}, t)=\sum_{n=1}^{N} \hat{b}_{n}(t) e^{I \boldsymbol{k}_{n}(t) \cdot \boldsymbol{x}},
\end{aligned}
$$

with the time-dependent wavevectors

$$
\boldsymbol{k}_{n}(t)=\left[\begin{array}{c}
k_{x}^{n} \\
k_{y}^{0, n}-S t k_{x}^{n} \\
k_{z}^{n}
\end{array}\right], n=1 \cdots N .
$$

The initial values of $k_{n}(0)=\left\|\boldsymbol{k}_{n}(0)\right\|$ lie in the interval $\left[k_{\min }, k_{\max }\right]=[1,100]$-defining some artificial Reynolds number $R e=\left(k_{\max } / k_{\min }\right)^{4 / 3} \approx 464$ - and are distributed according to the previously proposed density function ${ }^{32,36}$

$$
k_{n}(0)=k_{\min }\left(\frac{k_{\max }}{k_{\min }}\right)^{(n-1) /(N-1)}, n=1 \cdots N .
$$

For a given modulus $k_{n}^{0}=k_{n}(0)$ and random angles $\phi_{n}$ and $\delta_{n}^{0}=\delta_{n}(0)$, we construct the initial random wavevector

$$
\boldsymbol{k}_{n}^{0}=k_{n}^{0}\left[\begin{array}{c}
\cos \delta_{n}^{0} \cos \phi_{n} \\
\sin \delta_{n}^{0} \\
\cos \delta_{n}^{0} \sin \phi_{n}
\end{array}\right], n=1 \cdots N
$$

The random variable $\phi_{n}$ is uniformly distributed on $[0,2 \pi]$ while $\delta_{n}^{0}=\pi / 2-\operatorname{acos}\left(2 X_{n}-1\right)$ for $X_{n}$ uniformly distributed on $[0,1]$. An example of the corresponding wavectors spanning the unit sphere is shown in Figure 2(a). The initial complex amplitudes $\boldsymbol{u}_{n}(0)$ of the velocity field are distributed to satisfy the prescribed initial spectrum $E(k)=3 / 2 k^{-5 / 3}$

$$
\left\|\boldsymbol{u}_{n}^{0}\right\|=\sqrt{2 E\left(k_{n}^{0}\right) \Delta k_{n}}
$$

with

$$
\Delta k_{1}=\frac{1}{2}\left(k_{\min }\left(\frac{k_{\max }}{k_{\min }}\right)^{\frac{1}{N-1}}-k_{\min }\right),
$$




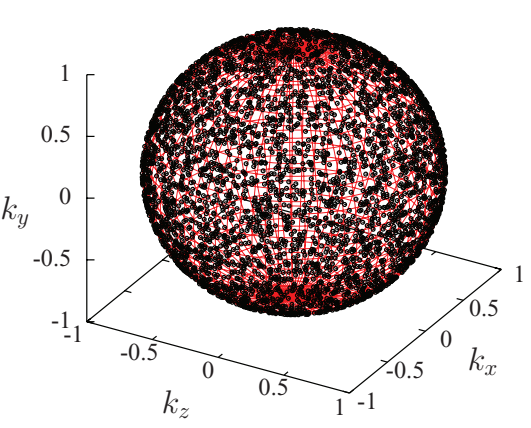

(a)

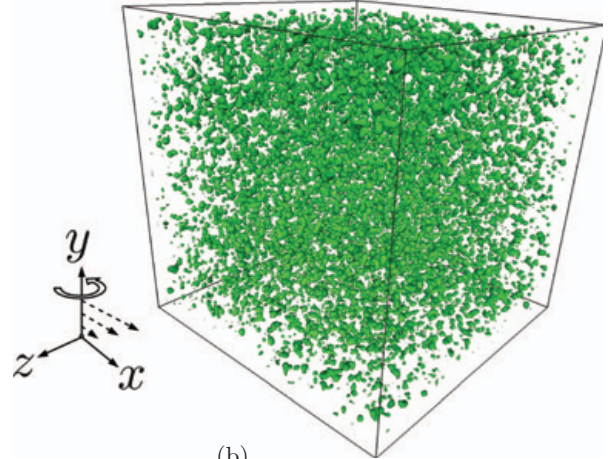

(b)

FIG. 2. (a) Initial distribution of $N=1024$ random wavevectors on the unit sphere. (b) The corresponding initial enstrophy distribution in the computational cube $[0,2 \pi]^{3}$, isosurfaces at $20 \%$ of the maximal enstrophy.

$$
\begin{aligned}
\Delta k_{N} & =\frac{1}{2}\left(k_{\min }\left(\frac{k_{\max }}{k_{\min }}\right)-k_{\min }\left(\frac{k_{\max }}{k_{\min }}\right)^{\frac{N-2}{N-1}}\right), \\
\Delta k_{j} & =\frac{1}{2}\left(k_{\min }\left(\frac{k_{\max }}{k_{\min }}\right)^{\frac{j+1}{N-1}}-k_{\min }\left(\frac{k_{\max }}{k_{\min }}\right)^{\frac{j-1}{N-1}}\right), j=2 \cdots N-1 .
\end{aligned}
$$

The distribution of enstrophy corresponding to this initial field is shown in Figure 2(b). The initial buoyancy field is set to zero, i.e.,

$$
\hat{b}_{n}(0)=0 \text { for } n=1 \cdots N
$$

so that the initial potential vorticity distribution is non-zero and results only in the kinematic induced potential vorticity

$$
\boldsymbol{\omega} \cdot \nabla \bar{b}(t=0)=\sum_{n=1}^{N} I\left(\boldsymbol{k}_{n}(0) \times \hat{\boldsymbol{u}}_{n}(0)\right) \cdot \nabla \bar{b} e^{I \boldsymbol{k}_{n}(0) \cdot \boldsymbol{x}} .
$$

The vortex admixture in the initial perturbation is therefore

$$
\frac{\|\omega \cdot \nabla \bar{b}\|}{N^{2}\|\omega\|}(0) \simeq \frac{\|\nabla \bar{b}\|}{N^{2}}=\sqrt{1+\varepsilon_{B}^{2}} .
$$

Once these initial conditions are set, Eqs. (15) are discretized in time with an implicit second order backward differentiation scheme for inconditional stability. Accordingly, time-marching is done in the Craya-Herring frame to enforce incompressibility. We present numerical results for both the complete solution of the nonlinear equations by DNS and by synthetic linear simulations (KS) of unstable baroclinic turbulence. Numerical details on the DNS code can be found in Pieri et al. ${ }^{4}$ suffices to say that it is a standard pseudo-spectral code based on the Rogallo method, ${ }^{7}$ with third-order time-stepping and complete desaliasing. Regarding the structure of the turbulent flow, low Reynolds number direct numerical simulations are in qualitative agreement with the linear approach. Figure 3(a) presents the spatial distribution of the enstrophy defined as

$$
\mathcal{E}(x)=\frac{1}{2} w(x) \cdot w(x) .
$$

In the unstable case, the flow becomes quasi-2D in the plane $(y, z)$. It means that quantities are invariant in the streamwise direction, i.e., that modes at $k_{x}=0$ are dominant, thus confirming the previous analytical results. In the classical hydrodynamic stability approach nonlinearities are quantified by the ratio of the amplitude of the nonlinear disturbance to a characteristic linear velocity. 


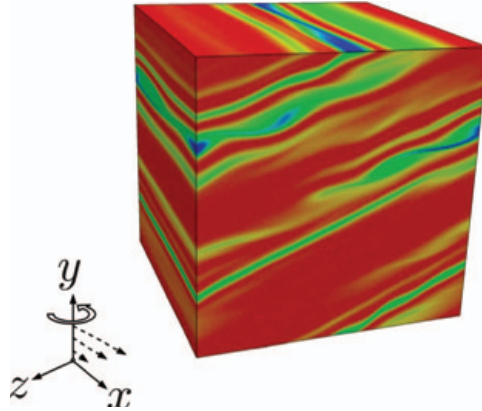

(a)
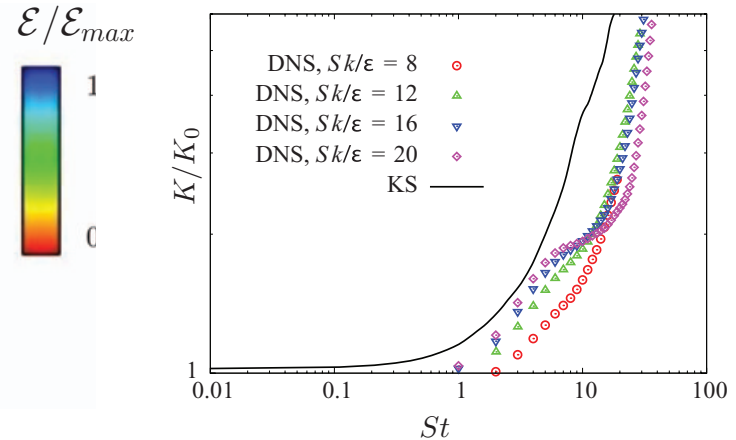

(b)

FIG. 3. Enstrophy distribution at time $S t=39$ in the unstable case at Richardson number $R i=0.8$ and baroclinicity parameter $\varepsilon_{B}=0.4$, corresponding to isopycnals oriented at 21.8 degrees. (a) Direct numerical simulation with a resolution of 192 $\times 288 \times 192$ at Reynolds number $R e=100$. (b) Comparison of the kinetic energy growth rate obtained by kinematic simulation with $N=12160$ random Fourier modes and 10 events at the same Reynolds number. The kinetic energy spectra used in the two computations are taken identical. Note that the KS model includes viscosity only for this comparison. See Appendix B for details about the introduction of viscosity in KS.

Since, in our case, the mean velocity field is prescribed only through its gradient, there is no obvious natural mean velocity scale available for the mean flow. However, the mean velocity gradient $S$ provides a time scale $1 / S$ which can be compared to the classical turbulent time-scale $K / \epsilon$. The ratio of these two time scales provides a substitute for the related amplitude parameter for the perturbation. Figure 3(b) presents the kinetic energy growth rate obtained by DNS for different values of the initial shear number $S^{*}=S K / \epsilon$ - often also called shear rapidity-and its comparison with KS. The shear number is defined as the ratio between a characteristic nonlinear time (eddy turn-over time) and a characteristic linear time, so that

$$
\begin{gathered}
S^{*}=\tau_{N L} / \tau_{L}, \\
\tau_{N L}=K / \epsilon, \\
\tau_{L}=1 / S .
\end{gathered}
$$

Higher values of $S^{*}$ correspond to more important linear effects. The results show that KS and DNS are in good agreement if the shear number is high enough, lets say $S^{*} \approx 10$, as highlighted also in the work by Jacobitz and Sarkar. ${ }^{37}$

\section{TRANSIENT GROWTH AND $k_{x} \approx 0$ MODES: INVESTIGATIONS BY RDT AND KS}

We investigate the time evolution of modes for which $k_{x} \approx 0$, and we consider Eq. (22) for the colatitude angle $\delta(t)$. We first observe that for modes $\boldsymbol{k}$ such that $\cos \phi \tan \delta_{0}>0$, there exists $\tau=\tan \delta_{0} /(S \cos \phi)>0$ such that for all $t \geq 0$

$$
\delta(t+\tau)=\operatorname{atan}(-t S \cos \phi) .
$$

Let us take $\phi=\frac{\pi}{2}+\varepsilon$ with $\varepsilon \ll 1$, that is modes close to $k_{x} \approx 0$. Then for small $\varepsilon, \tau$ is proportional to $1 / \varepsilon$. Numerical results showing the temporal behavior of the real part of the poloidal component $u^{(2)}$ of the velocity field are presented in Figure 4 for different values of $\varepsilon$. These modes are stable in that they eventually have a finite amplitude, but they present larger transient growth when $\varepsilon$ gets closer to zero, i.e., the amplitude of $\Re\left(u^{(2)}\right)$ increases from Figure 4(a) at $\varepsilon=0.01$ to Figure 4(c) at $\varepsilon$ $=0.001$. It is also shown that the time of change of regime is proportional to $1 / \varepsilon$, that is evolves as $\tau$. The change of regime is then experienced later the smaller $\varepsilon$. This particular behavior of modes with $k_{x} \rightarrow 0$ is confirmed by our kinematic simulation model (see Figure 5) that presents the distribution 

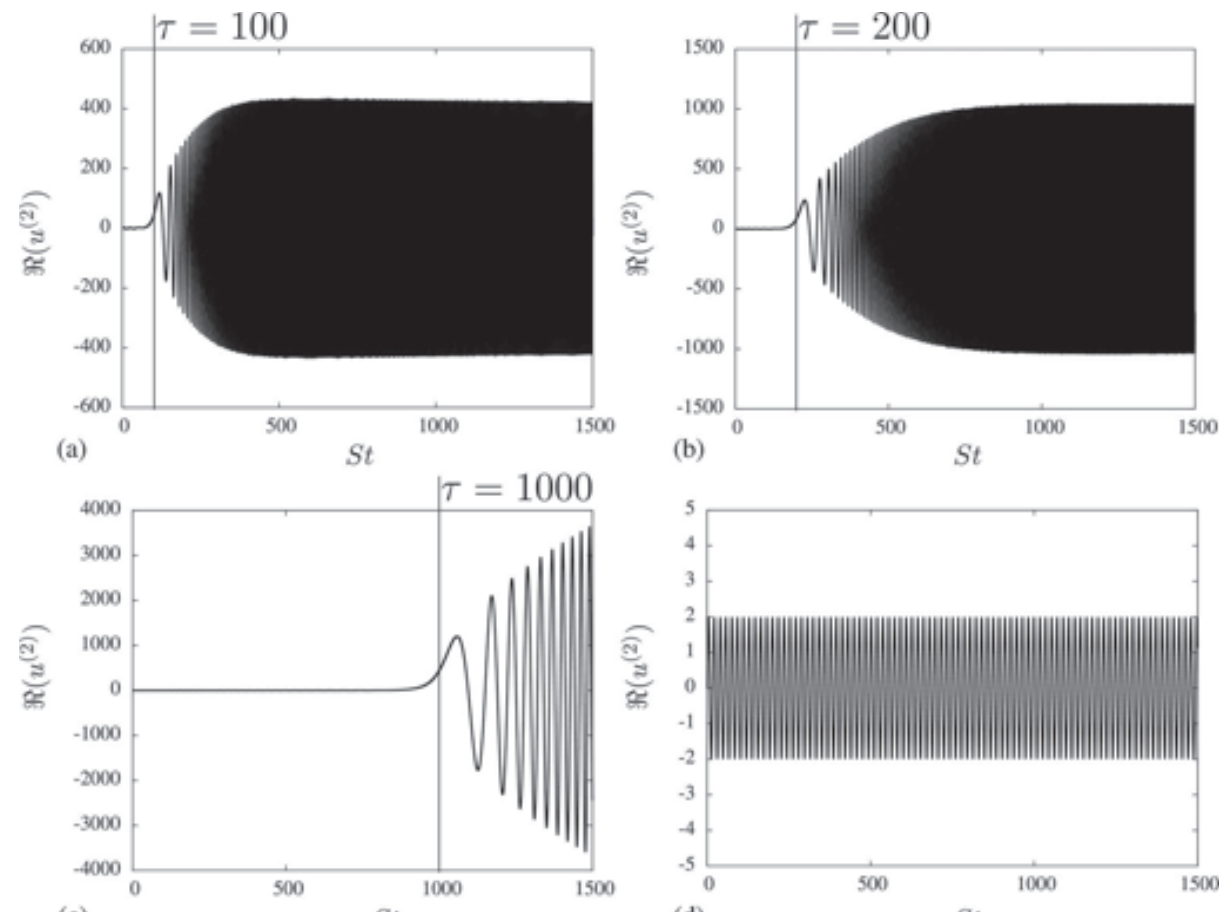

(c)

St.

(d)

St

FIG. 4. Real part of the poloidal component of the velocity with time $S t$ for $\phi=\frac{\pi}{2}+\varepsilon$ and $\delta=\frac{\pi}{4}$. (a) $\varepsilon=0.01$; (b) $\varepsilon=0.005$; (c) $\varepsilon=0.001$; (d) $\varepsilon=0$. Ri $=1$ and $\varepsilon_{B}=2$ with $u^{(3)}(0)=0$. RDT simulation for a single mode.
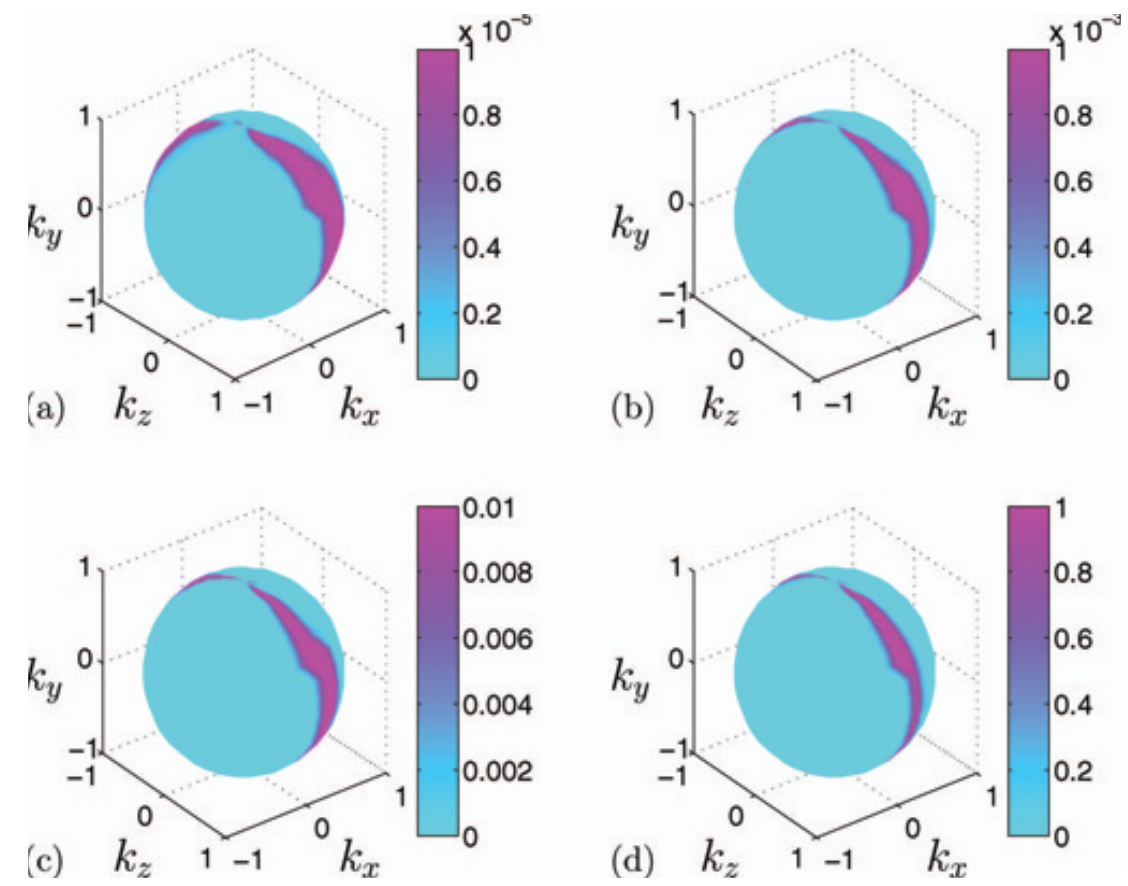

FIG. 5. Angular distribution of the kinetic energy on the initial sphere in spectral space for one realization with $48^{3}$ random Fourier modes. (a) $S t=50$; (b) $S t=100$; (c) $S t=150$; (d) $S t=200 . R i=0.25, \varepsilon_{B}=1$. 


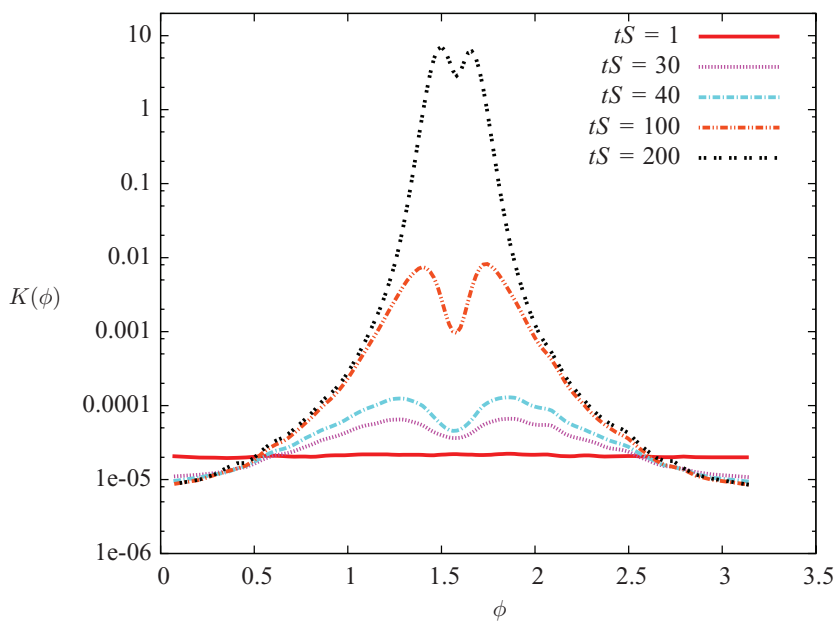

FIG. 6. Angular kinetic energy $K(\phi)$ defined by Eq. (43) as a function of the longitude $\phi \in[0, \pi]$. Kinematic Simulation results for $N=1024$ random modes averaged over 100 statistical events. $R i=0.5, \varepsilon_{B}=0.2$.

of kinetic energy over the unit sphere in spectral space, at four different times, for one particular set of initial conditions. We show that $k_{x} \approx 0$ modes play an important role at intermediate times, i.e., in the transition to baroclinic instability. At long times, the kinetic energy distribution match the analytical results ${ }^{1}$ and clearly demonstrate the dominance of modes with $k_{x}=0$, by the concentration of kinetic energy in the $k_{x}=0$ plane. Indeed, Figure 5 shows that the angular band containing high level of kinetic energy becomes narrower as $S t$ increase and are closer to $k_{x}=0$. It also highlights that the axisymmetry property is lost due to the presence of mean shear, meaning that kinetic energy density is not preserved by any rotation of the sphere.

A statistical average over 100 different initial conditions for the stochastic distribution of the modes in KS confirms this tendency, with results presented in Figure 6. We focus on the timeevolution of the longitudinal kinetic energy $K(\phi)$ defined as

$$
K(\phi)=\frac{1}{2} \int_{-\frac{\pi}{2}}^{\frac{\pi}{2}}\left(u^{(1)} u^{(1) *}+u^{(2)} u^{(2) *}\right)(\phi, \delta \cos \delta) d \delta .
$$

In addition to the previously mentioned concentration at $k_{x}=0$ at large time, Figure 6 for $t S=30, t S=40$ and $t S=100$ also shows two peaks of kinetic energy around $k_{x}=0$. At longer times, Figure 6 for $t S=200$ shows that these two peaks tend to merge into one centered on $k_{x}=0$ (or $\phi=\pi / 2$ ).

\section{A. Vortex-wave decomposition}

In order to better investigate the previous observations, we introduce a vortex-wave like decomposition following the survey by Sagaut and Cambon and other studies. ${ }^{21,38,39}$ In this decomposition, the flow is separated into a propagating part, the wave mode, and the part that carries potential vorticity, the vortical mode. In terms of Fourier amplitudes, and using the Craya-Herring modes, Ertel's theorem from Eq. (14) writes

$$
\left(k_{z}+R k_{y}\right) u^{(3)}-\left(\varepsilon_{B} \frac{k_{z} k_{y}}{k_{\perp}}+k_{\perp}\right) u^{(1)}-\varepsilon_{B} \frac{k_{x} k}{k_{\perp}} u^{(2)}=k_{\perp} u^{\left(\Omega_{p o t}\right)},
$$

with $k_{\perp}^{2}=k_{x}^{2}+k_{z}^{2}$. This result can be recovered by forming a linear combination of the three rows of the previous Kelvin-Moffatt matrix in Eq. (15). The simplified linear system one has to solve for 
a given mode in the Craya-Herring frame thus becomes

$$
\frac{d}{d t}\left[\begin{array}{l}
u^{(1)} \\
u^{(2)}
\end{array}\right]=S\left[\begin{array}{cc}
0 & f(\delta, \phi) \\
\frac{\cos ^{2} \delta \cos \phi \sin \delta(\sin \phi-R)}{f(\delta, \phi)} & \frac{\cos ^{2} \delta\left(\sin ^{2} \phi-R i\right)}{f(\delta, \phi)}-f(\delta, \phi)
\end{array}\right]\left[\begin{array}{c}
u^{(1)} \\
u^{(2)}
\end{array}\right]+\left[\begin{array}{c}
0 \\
-R i \cos ^{2} \delta
\end{array}\right] u^{\left(\Omega_{p o t}\right)},
$$

where

$$
f(\delta, \phi)=\cos \delta \sin \phi+R \sin \delta .
$$

The previous system of equations amounts to the vortex-wave (non-propagating/propagating) decomposition expressed in the Craya-Herring frame for baroclinic turbulence. The spectral velocity component $u^{\left(\Omega_{p o t}\right)}$ can be linked to the velocity components $\boldsymbol{u}_{\pi}$ from Eq. (11) in physical space in agreement with Staquet and Riley. ${ }^{30}$ This new formulation highlights first that vertical modes $\boldsymbol{k}$ with $\delta \approx \pm \pi / 2$ are not coupled with the PV mode $u^{\left(\Omega_{p o t}\right)}$ and secondly that the coupling is maximal for equatorial modes $\boldsymbol{k}$ having $\delta \approx 0$, where $\delta$ is time-dependent in our model. Equation (22) shows that $\delta \rightarrow \pm \pi / 2$ asymptotically at large time, describing that the shear dynamics tends to reduce the coupling with the PV mode. It also shows that the modes satisfying the condition $\cos \phi \tan \delta_{0}>0$ that were previously identified to be transitionally dominant are also the modes that experience an angle $\delta=0$, i.e., a maximal coupling with the PV mode at the critical time $\tau$ defined in Sec. II. This transitional growth of modes with $k_{x} \approx 0$ is thus the consequence of potential vorticity conservation and of the coupling with the PV mode enforced by shear effects at intermediate times only since Eq. (45) shows that, at long times, the shear inhibits the coupling with $u^{\left(\Omega_{p o t}\right)}$.

\section{B. Numerical results}

We present here numerical results that confirm the previous discussion of the role of the PV mode. RDT simulations are done with zero initial potential vorticity (called the Zero-Potential vorticity mode in Muller et al. ${ }^{39}$ or inertia-gravity mode without shear effects) to clearly show the modified dynamics arising from a lack of coupling with $u^{\left(\Omega_{p o t}\right)}$. Corresponding zero-PV modes are obtained by substracting the kinematic-induced potential vorticity with a careful choice of an appropriate initial condition for $u^{(3)}(0)$. Figure 7 shows the spatial distribution of this vortex mode highlighting that the crests lie on the isopycnal surfaces. The results without the coupling with the PV mode are presented in Figure 8, with the same parameters as in Figure 4. We observe a change of regime at the same characteristic time $\tau$ but $\mathfrak{R}\left(u^{(2)}\right)$ ) does not experience large variations in amplitude when reducing $\varepsilon$. In addition, these amplitudes take low values when the coupling is not activated, testifying that the mode's selection process is inhibated. It shows that the transient behavior of these modes is due to small $k_{x}$ but that a non-zero potential vorticity is necessary to
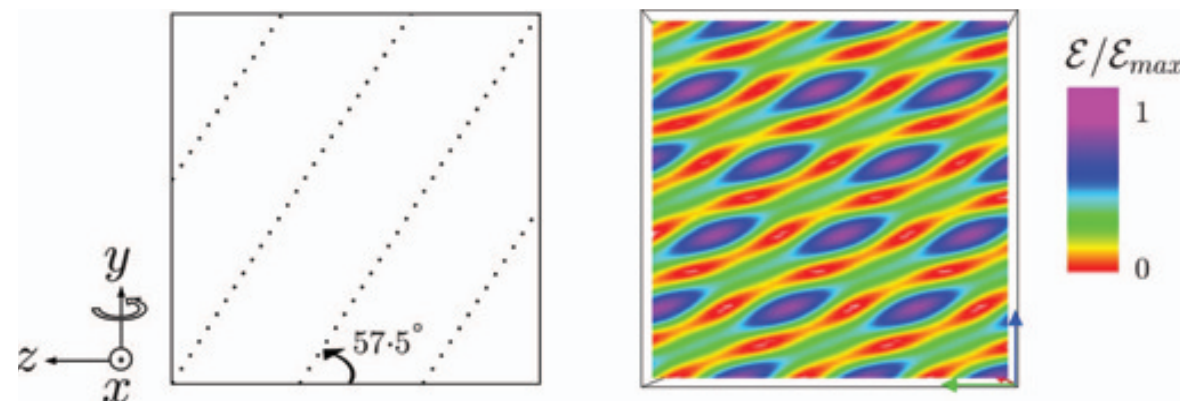

FIG. 7. Normalized enstrophy distribution at: $t S=700$ for $\phi=\frac{\pi}{2}+\varepsilon$ and $\delta=\frac{\pi}{4}$ with $\varepsilon=0.01$. $R i=1, \varepsilon_{B}=0.2$. No coupling with the vortex mode $u^{\left(\Omega_{p o t}\right)}=0$. RDT simulations for a single wave mode. The figure shows the orientation of creasts and troughs (regions colored in purple—-large dark cat's eyes and red—small dark cat's eyes,respectively) with an angle $\delta(t S=700) \approx 57.5$ degrees. 

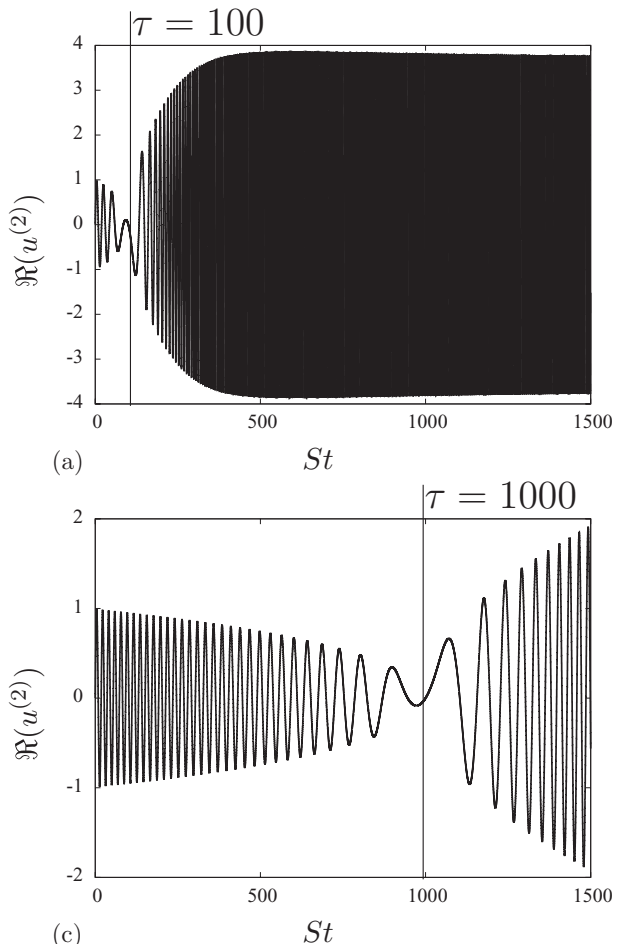

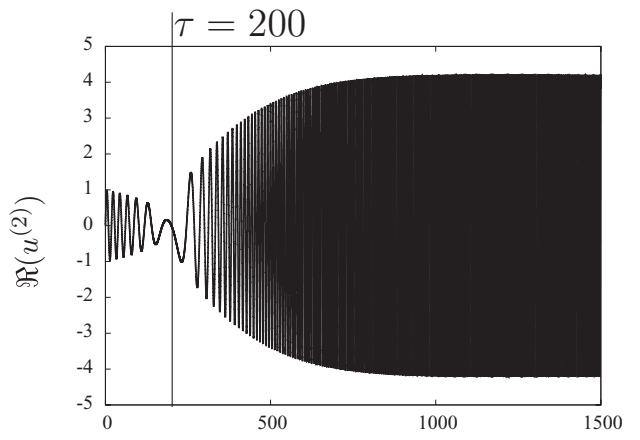

(b)

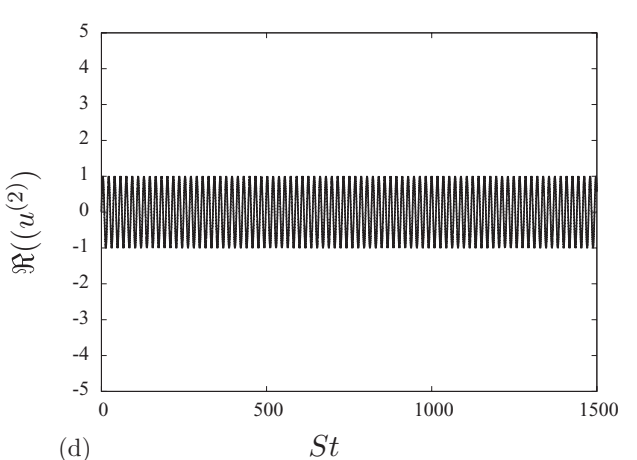

FIG. 8. Real part of the poloidal component of the velocity with time $S t$ for $\phi=\frac{\pi}{2}+\varepsilon$ and $\delta=\frac{\pi}{4}$. (a) $\varepsilon=0.01$; (b) $\varepsilon=0.005$; (c) $\varepsilon=0.001$; (d) $\varepsilon=0 . R i=1$ and $\varepsilon_{B}=2$ with $u^{\left(\Omega_{p o t}\right)}(0)=0$. RDT simulation for a single mode.

observe its temporary dominance. The transition regime at $t \in[0, \tau]$ corresponds to the passage from small amplitudes to larger ones. During this regime, the poloidal velocity component behaves like the product of an exponential damping term by a destabilizing exponential term if the coupling with PV is activated (for details, refer to Appendix C, Eq. (C4)). It shows that, when $u^{\left(\Omega_{p o t}\right)} \neq 0$, the poloidal part has an amplitude proportional to $1 / \sqrt{\varepsilon}$. This completes our analysis and demonstrates that modes closer to $k_{x}=0$ will experience higher transitional growth at longer times $\tau$ (see Figure 4).

\section{CONCLUSION}

In this paper we have shown that vortex-wave decomposition helps to interpret the transitional stability regime in the baroclinic context. The coupling with the vortex mode, noted $u^{\left(\Omega_{p o t}\right)}$ here, is responsible for high transitional growth of modes with $k_{x} \approx 0$. It gives a complementary analysis to the work done by Ref. 5. Rapid distortion theory and Kinematic Simulation completed by analytical work are all consistent with this new result. In particular, in order to observe an important transitional growth, it is necessary to satisfy both $k_{x} \approx 0$ but $k_{x} \neq 0$ and $u^{\left(\Omega_{p o t}\right)} \neq 0$. The importance of the initial "vortex" admixture to get transient growth is then of capital interest. The extension of these results to the nonlinear case can be done in the limit of high shear number $S^{*}$ $=S K / \epsilon$ and sufficiently large scales. Indeed for the small scales-that are also the least energetic scales - the initial adaptation of turbulence will cause a growth of kinetic energy that has nothing to do with linear effects but is more linked to nonlinear energy cascade processes. The present linear numerical model for baroclinic turbulence (referred to as KS in the paper)-and its ability to enforce potential vorticity conservation - could be well-suited for geophysical applications. In particular its use as a subgrid-scale model in geophysical codes should be investigated. This work will be completed by complementary DNS. First, the upper stability bound $R i=1$ recalled in 
Sec. IV A proposed by Salhi and Cambon ${ }^{1}$ needs to be compared to limits obtained by nonlinear simulations. A stability analysis at low resolution is in progress. Second, the role played by the (nonlinear) potential vorticity in DNS and its consequence on turbulence dynamics should lead to new research.

\section{APPENDIX A: ANALYTICAL RESULTS FOR RDT IN PHYSICAL AND FOURIER SPACE}

\section{Rapid distortion theory equations in physical space}

We assume the following mean background velocity gradient:

$$
\frac{\partial u_{i}}{\partial x_{j}}=\left[\begin{array}{ccc}
0 & 0 & 0 \\
S & 0 & 0 \\
0 & 0 & 0
\end{array}\right],
$$

where $S$ accounts for the uniform mean shear. The mean background density gradient is given by the geostrophic adjustment as

$$
\bar{b}=N^{2} y+\varepsilon_{B} N^{2} z
$$

Denoting the fluctuating velocity (resp. buoyancy) fields by $\boldsymbol{u}^{\prime}$ (resp. $b^{\prime}$ ) we have in physical space:

$$
\begin{aligned}
\frac{\partial u_{i}^{\prime}}{\partial t}+S y \frac{\partial u_{i}^{\prime}}{\partial x}+S \delta_{i x} u_{y}+f \epsilon_{i y j} u_{j}^{\prime}+\frac{1}{\rho_{0}} \frac{\partial p^{\prime}}{\partial x_{i}} & =b^{\prime} \delta_{i y}, \\
\frac{\partial b^{\prime}}{\partial t}+S y \frac{\partial b^{\prime}}{\partial x} & =-N^{2}\left(u_{y}^{\prime}+\varepsilon_{B} u_{z}^{\prime}\right), \\
\frac{\partial u_{i}^{\prime}}{\partial x_{i}} & =0,
\end{aligned}
$$

where $\epsilon_{i j k}$ is the Levi-Civita tensor. These are the linearized Boussinesq equations in physical space. In Sec. 2 of Appendix A, we introduce the Fourier decomposition for the fluctuating fields and derive the RDT equations in the standard spectral space and in the Craya-Herring frame.

\section{RDT equations in spectral space: Fixed frame and Craya-Herring frame}

We start from a normal modes decomposition like

$$
\begin{aligned}
\boldsymbol{u}^{\prime}(t) & =\hat{\boldsymbol{u}}(t) e^{i \boldsymbol{k}(t) \cdot \boldsymbol{x}}, \\
b^{\prime}(t) & =\hat{b}(t) e^{i \boldsymbol{k}(t) \cdot \boldsymbol{x}} .
\end{aligned}
$$

Under the effect of shear, this decomposition is valid only when assuming time-dependent wavevectors $\boldsymbol{k}(t)$. Moving with the Lagrangian frame of the mean background flow, the vertical components $k_{y}$ of the wavevectors are advected as

$$
\frac{\partial k_{i}}{\partial t}+\frac{\partial u_{j}}{\partial x_{i}} k_{j}=0
$$

and the incompressibility condition can be expressed as

$$
\hat{u}_{i} k_{i}=0 \text {. }
$$

Injecting the normal mode decomposition in Eqs. (A2)-(A4) and applying the projector $P_{i j}=\delta_{i j}$ $-k_{i} k_{j} / k^{2}$ to get rid of pressure terms, we obtain:

$$
\begin{aligned}
\frac{d \hat{u}_{i}}{d t} & =-\left[S \delta_{j y}\left(\delta_{i x}-2 \frac{k_{i} k_{x}}{k^{2}}\right)+2 \Omega P_{i n} \epsilon_{n y j}\right] \hat{u}_{j}+P_{i y} \hat{b}, \\
\frac{d \hat{b}}{d t} & =-N^{2} \hat{u}_{y}-N^{2} \varepsilon_{B} \hat{u}_{z},
\end{aligned}
$$


in the fixed cartesian spectral frame. To reduce the number of variables, we project these equations onto the solenoidal Craya-Herring frame of which a basis is

$$
\begin{aligned}
\boldsymbol{e}^{(1)} & =\frac{\boldsymbol{k} \times \boldsymbol{n}}{\|\boldsymbol{k} \times \boldsymbol{n}\|}, \\
\boldsymbol{e}^{(2)} & =\frac{\boldsymbol{k}}{\|\boldsymbol{k}\|} \times \frac{\boldsymbol{k} \times \boldsymbol{n}}{\|\boldsymbol{k} \times \boldsymbol{n}\|}, \\
\boldsymbol{e}^{(3)} & =\frac{\boldsymbol{k}}{\|\boldsymbol{k}\|},
\end{aligned}
$$

where $\boldsymbol{e}^{(1)}$ is the toroidal direction and $\boldsymbol{e}^{(2)}$ the poloidal direction. In the case where $\boldsymbol{y}$ denotes the vertical direction, it gives the following transformation matrix

$$
\mathcal{P}_{\mathcal{B} \rightarrow \mathcal{C H}}=\left[\begin{array}{ccc}
-\frac{k_{z}}{k_{\perp}} & \frac{k_{x} k_{y}}{k k_{\perp}} & \frac{k_{x}}{k} \\
0 & -\frac{k_{\perp}}{k} & \frac{k_{y}}{k} \\
\frac{k_{x}}{k_{\perp}} & -\frac{k_{y} k_{z}}{k k_{\perp}} & \frac{k_{z}}{k}
\end{array}\right] .
$$

The incompressibility condition is then satisfied when $\hat{\boldsymbol{u}}(\boldsymbol{k}) \cdot \boldsymbol{e}^{(3)}=0$. To pass from the equations in fixed frame of reference (A9) and (A10) to its equivalent formulation in Craya-Herring frame, one has to take into account the possible time-dependency of the unit vectors $\left(\boldsymbol{e}^{(1)}, \boldsymbol{e}^{(2)}\right)$ :

$$
\begin{aligned}
\frac{d u^{(1)}}{d t} & =\boldsymbol{e}^{(1)} \cdot \frac{d \hat{\boldsymbol{u}}}{d t}+\hat{\boldsymbol{u}} \cdot \frac{d \boldsymbol{e}^{(1)}}{d t}, \\
\frac{d u^{(2)}}{d t} & =\boldsymbol{e}^{(2)} \cdot \frac{d \hat{\boldsymbol{u}}}{d t}+\hat{\boldsymbol{u}} \cdot \frac{d \boldsymbol{e}^{(2)}}{d t} .
\end{aligned}
$$

However, because the Craya-Herring frame is orthonormal we have

$$
\begin{aligned}
& \frac{d \boldsymbol{e}^{(1)}}{d t} \cdot \boldsymbol{e}^{(1)}=0, \\
& \frac{d \boldsymbol{e}^{(2)}}{d t} \cdot \boldsymbol{e}^{(2)}=0,
\end{aligned}
$$

and

$$
\frac{d \boldsymbol{e}^{(2)}}{d t} \cdot \boldsymbol{e}^{(1)}=-\frac{d \boldsymbol{e}^{(1)}}{d t} \cdot \boldsymbol{e}^{(2)}
$$

In the particular case of a pure shear flow with vertical polar axis $n$

$$
\frac{d e^{(1)}}{d t}=0
$$

Finally the above equation simplifies the system so that

$$
\hat{\boldsymbol{u}} \cdot \frac{d \boldsymbol{e}^{(2)}}{d t}=0
$$

and

$$
\hat{\boldsymbol{u}} \cdot \frac{d \boldsymbol{e}^{(1)}}{d t}=0
$$

Thus we get the reduced linear system

$$
\begin{aligned}
& \frac{d u^{(1)}}{d t}=S \frac{k_{z}+\varepsilon_{B} R i k_{y}}{k} u^{(2)}, \\
& \frac{d u^{(2)}}{d t}=-S \varepsilon_{B} R i \frac{k_{y}}{k} u^{(1)}+S \frac{k_{x} k_{y}}{k^{2}} u^{(2)}-S R i \frac{k_{\perp}}{k} u^{(3)},
\end{aligned}
$$




$$
\frac{d u^{(3)}}{d t}=-S \varepsilon_{B} \frac{k_{x}}{k_{\perp}} u^{(1)}+S\left(\frac{k_{\perp}}{k}+\varepsilon_{B} \frac{k_{z} k_{y}}{k k_{\perp}}\right) u^{(2)},
$$

where $u^{(3)}$ is the normalized buoyancy defined as

$$
u^{(3)}=\frac{S}{N^{2}} \hat{b} \text {. }
$$

\section{APPENDIX B: VISCOUS KINEMATIC SIMULATION: A ROAD TOWARD SUBGRID-SCALE MODELLING}

Consider the linear system coming form the previous inviscid RDT analysis in the Craya-Herring frame for a given wave vector $\boldsymbol{k}$

$$
\begin{aligned}
\frac{d \hat{\boldsymbol{u}}_{\boldsymbol{k}}^{\nu=0}}{d t} & =A(\boldsymbol{k}) \hat{\boldsymbol{u}}_{\boldsymbol{k}}^{\nu=0}, \\
\hat{\boldsymbol{u}}_{\boldsymbol{k}}^{\nu=0} & =\left(u_{\boldsymbol{k}}^{(1)}, u_{\boldsymbol{k}}^{(2)}, u_{\boldsymbol{k}}^{(3)}\right)^{T, \nu=0},
\end{aligned}
$$

where

$$
\left(u_{\boldsymbol{k}}^{(1)}, u_{\boldsymbol{k}}^{(2)}, u_{\boldsymbol{k}}^{(3)}\right)^{T, \nu=0}=\mathcal{P}_{\mathcal{B} \rightarrow \mathcal{C H}} \cdot\left(\hat{u}_{\boldsymbol{k}, x}, \hat{u}_{\boldsymbol{k}, y}, \hat{u}_{\boldsymbol{k}, z}\right)^{T, \nu=0}+\frac{S}{N^{2}} \hat{b}_{\boldsymbol{k}}^{\nu=0} \boldsymbol{e}_{3} .
$$

Let $\left(\hat{u}_{k, x}, \hat{u}_{k, y}, \hat{u}_{k, z}, \hat{b}_{k}\right)^{T}$ be the viscous spectral solution. We introduce the kinematic viscosity $v$ $>0$ and the Prandtl number $\operatorname{Pr}=v / \chi$ is set to $P r=1$ without lost of generality. The viscous linear solution is then obtained easily as

$$
\left(\hat{u}_{k, x}, \hat{u}_{k, y}, \hat{u}_{\hat{k}, z}, \hat{b}_{\boldsymbol{k}}\right)^{T}=c_{\boldsymbol{k}, v}(t)\left(\hat{u}_{\boldsymbol{k}, x}, \hat{u}_{\boldsymbol{k}, y}, \hat{u}_{\boldsymbol{k}, z}, \hat{b}_{\boldsymbol{k}}\right)^{T, \nu=0},
$$

with

$$
c_{\boldsymbol{k}, v}(t)=\exp \left[-v \int_{0}^{t} k^{2}(\tau) \mathrm{d} \tau\right]=\exp \left[-v\left\{k^{2} t-S t^{2} k_{x} k_{z}+\frac{S^{2}}{3} t^{3} k_{x}^{2}\right\}\right] .
$$

Note that all the coefficients involved in Eq. (B5) are taken at time $t=0$ so that there is no time dependence in these coefficients. Now, the viscous kinematic simulation model is obtained by multiplying each inviscid single mode solution $\hat{\boldsymbol{u}}_{\boldsymbol{k}}^{v=0}(t)$ by its corresponding coefficient $c_{\boldsymbol{k}, v}(t)$ :

$$
\begin{aligned}
& \boldsymbol{u}(\boldsymbol{x}, t)=\sum_{n=1}^{N} c_{\boldsymbol{k}_{n}, v}(t) \hat{\boldsymbol{u}}_{n}^{\nu=0}(t) e^{I \boldsymbol{k}_{n}(t) \cdot \boldsymbol{x}}, \\
& b(\boldsymbol{x}, t)=\sum_{n=1}^{N} c_{\boldsymbol{k}_{n}, v}(t) \hat{b}_{n}^{\nu=0}(t) e^{I \boldsymbol{k}_{n}(t) \cdot \boldsymbol{x}} .
\end{aligned}
$$

The implementation of such a kinematic simulation model for subgrid scales modelling has been discussed by Flohr and Vassilicos. ${ }^{31}$ Coupling of the inviscid model with LES should be studied further. Its ability to impose potential vorticity conservation-at small scales in the subgrid-scale model-may have interesting applications for geophysics. Note that even if the amplitude of an inviscid solution does not depend on the modulus of the wavevector $\boldsymbol{k}$, using in KS a spectrum that spreads over some decades of $k$ allows to introduce a range of different scales. This is equivalent, from a theoretical point of view, to setting an artificial Reynolds number $R e$ based on the ratio of the scales involved in the energy spectrum

$$
\operatorname{Re} \propto\left(\frac{k_{\max }}{k_{\min }}\right)^{4 / 3} .
$$


In the viscous case, viscosity yields a physical dissipative length scale so that, if $k_{p}$ denotes the wavenumber for which the energy spectrum is maximum, one may use

$$
\operatorname{Re} \propto \frac{S}{\nu k_{p}^{2}} .
$$

\section{APPENDIX C: BEHAVIOR OF $\boldsymbol{k}_{\boldsymbol{x}} \approx 0$ MODES}

We study the case of modes around $k_{x}=0$ for a Richardson number $R i \approx 1$. We approximate the solution of the reduced linear system presented in Sec. VI by Eq. (45). The analysis is done at the first order in $\varepsilon$. We recall that $\varepsilon$ stands for a small angle perturbation around $\pi / 2$, such that $\phi=\pi / 2+\varepsilon$. To first order we get

$$
\begin{gathered}
f(\delta, \phi) \approx 1+R t S \varepsilon+o(\varepsilon), \\
\frac{d}{d t}\left[\begin{array}{l}
u^{(1)} \\
u^{(2)}
\end{array}\right]=S\left[\begin{array}{cc}
0 & 1+R t S \varepsilon \\
0-R i+R(R i-2) t S \varepsilon
\end{array}\right]\left[\begin{array}{l}
u^{(1)} \\
u^{(2)}
\end{array}\right]+\left[\begin{array}{c}
0 \\
-R i
\end{array}\right] u^{\left(\Omega_{p o t}\right)}+o(\varepsilon) .
\end{gathered}
$$

The equation for $u^{(2)}$ is

$$
\frac{d u^{(2)}}{d t}(t+\tau)=-\left[S R i+2 \frac{S^{2} \varepsilon R}{2}(2-R i) t\right] u^{(2)}(t+\tau)-R i u^{\left(\Omega_{p o t}\right)},
$$

where $\tau$ is defined by Eq. (42) in Sec. VI. The general solution of this equation is

$$
u^{(2)}(t+\tau)=\left[-\frac{\operatorname{Ri}^{\left(\Omega_{p o t}\right)}}{\alpha} \frac{\sqrt{\pi}}{2}(\operatorname{Erfi}(\alpha t+\beta)-\operatorname{Erfi}(\beta))+u^{(2)}(\tau) e^{\beta^{2}}\right] e^{-(\alpha t+\beta)^{2}},
$$

with

$$
\begin{aligned}
& \alpha=S \sqrt{\frac{\varepsilon R}{2}(2-R i)} \propto \frac{1}{\sqrt{\tau}}, \\
& \beta=\frac{S R i}{2 \alpha} \propto \sqrt{\tau},
\end{aligned}
$$

and where Erfi is the complex error function defined by

$$
\operatorname{Erfi}(z)=\frac{2}{\sqrt{\pi}} \int_{0}^{z} e^{\zeta^{2}} \mathrm{~d} \zeta
$$

Equation (C4) shows that for $u^{\left(\Omega_{p o t}\right)}=0$ we have a damped stable solution $u^{(2)}$ while for non-zero $u^{\left(\Omega_{p o t}\right)}$ the damping term is coupled to the vortex mode by an Erfi function, allowing dramatic transient growth of $u^{(2)}$. The reader will note that when $R i>2$, the coefficient $\alpha$ becomes complex. However, if one looks at the modes $\phi=\pi / 2-\varepsilon, \alpha$ remains real and the same analysis is true for $R i>2$.

${ }^{1}$ A. Salhi and C. Cambon, "Advances in rapid distortion theory: From rotating shear flows to the baroclinic instability," J. Appl. Mech. 73(3), 449-460 (2006).

${ }^{2}$ C. G. Rossby, "On the mutual adjustment of pressure and velocity distributions in certain simple current systems," J. Mar. Res. 1, 239-263 (1938).

${ }^{3}$ J. Pedlosky, "The nonlinear dynamics of baroclinic wave ensembles," J. Fluid Mech. 102, 169-209 (1981).

${ }^{4}$ A. Pieri, "Homogeneous turbulence dynamics in the baroclinic context," Ph.D. dissertation (École centrale de Lyon, France, 2012).

${ }^{5}$ G. R. Mamatsashvili, V. S. Avsarkisov, G. D. Chagelishvili, R. G. Chanishvili, and M. V. Kalashnik, "Transient dynamics of nonsymmetric perturbations versus symmetric instability in baroclinic zonal shear flows," J. Atmos. Sci. 97, 2972-2989 (2010).

${ }^{6}$ A. Salhi, T. Lehner, F. Godeferd, and C. Cambon, "Magnetized stratified rotating shear waves," Phys. Rev. E 85(2), 026301 (2012).

${ }^{7}$ R. S. Rogallo, "Numerical experiments in homogeneous turbulence numerical experiments in homogeneous turbulence," Nasa Technical Memorandum, 1981 
${ }^{8}$ S. E. Holt, J. R. Koseff, and J. H. Ferziger, "A numerical study of the evolution and structure of homogeneous stably stratified sheared turbulence,” J. Fluid Mech. 237, 499-539 (1992).

${ }^{9}$ F. G. Jacobitz, S. Sarkar, and C. W. van Atta, "Direct numerical simulations of the turbulence evolution in a uniformly sheared and stably stratified flow,” J. Fluid Mech. 342(1), 231-261 (1997).

${ }^{10}$ T. Gerz, U. Schumann, and S. E. Elghobashi, "Direct numerical simulation of stratified homogeneous turbulent shear flows,” J. Fluid Mech. 200, 563-594 (1989).

${ }^{11}$ H.-J. Kaltenbach, T. Gerz, and U. Schumann, "Large-eddy simulation of homogeneous turbulence and diffusion in stably stratified shear flow," J. Fluid Mech. 280, 1-40 (1994).

${ }^{12} \mathrm{~K}$. H. Keller and C. W. Van Atta, "An experimental investigation of the vertical temperature structure of homogeneous stratified shear turbulence,” J. Fluid Mech. 425, 1-29 (2000).

${ }^{13}$ P. Piccirillo and C. W. Van Atta, "The evolution of a uniformly sheared thermally stratified turbulent flow," J. Fluid Mech. 334, 61-86 (1997)

${ }^{14}$ H. Hanazaki and J. C. R. Hunt, "Structure of unsteady stably stratified turbulence with mean shear," J. Fluid Mech. 507, $1-42$ (2004).

${ }^{15}$ A. Deloncle, J.-M. Chomaz, and P. Billant, "Three-dimensional stability of a horizontally sheared flow in a stably stratified fluid," J. Fluid Mech. 570, 297-305 (2007).

${ }^{16}$ J. W. Miles, "On the stability of heterogeneous shear flows," J. Fluid Mech. 10(04), 496-508 (1961).

${ }^{17}$ L. N. Howard, "Note on a paper of John W. Miles," J. Fluid Mech. 10(04), 509-512 (1961).

${ }^{18}$ G. Simon, "Dynamique multi-échelle en fluide stratifié tournant, instabilité de cisaillement et cyclone intense," Ph.D. dissertation, Ecole Centrale de Lyon, 2007.

${ }^{19}$ L. N. Trefethen and M. Embree, Spectra and Pseudospectra: The Behavior of Nonnormal Matrices and Operators (Princeton University, 2005).

${ }^{20}$ This is relevant when the mean shear is induced by differential rotation of the flow around its spanwise direction.

${ }^{21}$ A. G. Tevzadze, G. D. Chagelishvili, J. P. Zahn, R. G. Chanishvili, and J. G. Lominadze, "On hydrodynamic shear turbulence in stratified Keplerian disks: Transient growth of small-scale 3D vortex mode perturbations,” Astron. Astrophys. 407(3), 779-786 (2003)

${ }^{22}$ H. K. Moffatt, "The interaction of turbulence with strong wind shear," in Proceedings of the URSI-IUGG International Colloquium on 'Atmospheric Turbulence and Radio Wave Propagation' Moscow, June 1965, edited by A. M. Yaglom and V. I. Tatarsky (Nauka, Moscow, 1967), pp. 139-154.

${ }^{23}$ J. C. H. Fung, J. C. R. Hunt, N. A. Malik, and R. J. Perkins, "Kinematic simulation of homogeneous turbulence by unsteady random Fourier modes,” J. Fluid Mech. 236(1), 281-318 (1992).

${ }^{24}$ G. D. Chagelishvili, A. G. Tevzadze, G. Bodo, and S. S. Moiseev, "Linear mechanism of wave emergence from vortices in smooth shear flows," Phys. Rev. Lett. 79(17), 3178-3181 (1997).

${ }^{25}$ M. Timoumi and A. Salhi, "Equilibrium states in homogeneous turbulence with baroclinic instability," Theor. Comput. Fluid Dyn. 23, 353-374 (2009).

${ }^{26}$ G. K. Batchelor, "Heat convection and buoyancy effects in fluids," Q. J. R. Meteorol. Soc. 80(345), 339-358 (1954).

${ }^{27}$ J. S. Turner, Buoyancy Effects in Fluids (Cambridge University Press, 1980).

${ }^{28}$ H. Ertel, "Ein neuer hydrodynamischer wirbelsatz," Meteorol. Z. 59, 271-281 (1942).

${ }^{29}$ C. G. Rossby, "Planetary flow patterns in the atmosphere," Q. J. R. Meteorol. Soc. 66, 68-87 (1940).

${ }^{30}$ C. Staquet and J. J. Riley, "On the velocity field associated with potential vorticity," Dyn. Atmos. Oceans 14, 93-123 (1989).

${ }^{31}$ P. Flohr and J. C. Vassilicos, "A scalar subgrid model with flow structure for large-eddy simulations of scalar variances," J. Fluid Mech. 407(1), 315-349 (2000).

${ }^{32}$ N. A. Malik, "Studies in turbulent dispersion using kinematic simulation," Ph.D. dissertation (St. Edmund's College, University of Cambridge, 1991).

${ }^{33}$ N. A. Malik and J. C. Vassilicos, "A lagrangian model for turbulent dispersion with turbulent-like flow structure: Comparison with direct numerical simulation for two-particle statistics," Phys. Fluids 11, 1572 (1999).

${ }^{34}$ D. M. Lewis and T. J. Pedley, "Planktonic contact rates in homogeneous isotropic turbulence: Theoretical predictions and kinematic simulations," J. Theor. Biol. 205(3), 377-408 (2000).

${ }^{35}$ C. Cambon, F. S. Godeferd, F. C. G. A. Nicolleau, and J. C. Vassilicos, "Turbulent diffusion in rapidly rotating flows with and without stable stratification," J. Fluid Mech. 499, 231-255 (2004).

${ }^{36}$ S. Goto, D. R. Osborne, J. C. Vassilicos, and J. D. Haigh, "Acceleration statistics as measures of statistical persistence of streamlines in isotropic turbulence," Phys. Rev. E 71(1), 015301 (2005).

${ }^{37}$ F. G. Jacobitz and S. Sarkar, "On the shear number effect in stratified shear flow," Theor. Comp. Fluid Dyn. 13(3), 171-188 (1999).

${ }^{38}$ P. Sagaut and C. Cambon, Homogeneous Turbulence Dynamics (Cambridge University Press, 2008).

${ }^{39} \mathrm{P}$. Müller, "Ertel's potential vorticity theorem in physical oceanography," Rev. Geophys. 33, 67-97, doi:10.1029/94RG03215 (1995). 